\title{
Computing Equilibria in General Equilibrium Models via Interior-point Methods
}

\author{
MERCEDES ESTEBAN-BRAVO \\ Department of Computing, Imperial College of Science, Technology and Medicine, 180 Queen's \\ Gate, London,U.K.; E-mail:meb@doc.ic.ac.uk
}

(Accepted 11 December 2002)

\begin{abstract}
In this paper we study new computational methods to find equilibria in general equilibrium models. We first survey the algorithms to compute equilibria that can be found in the literature on computational economics and we indicate how these algorithms can be improved from the computational point of view. We also provide alternative algorithms that are able to compute the equilibria in an efficient manner even for large-scale models, based on interior-point methods. We illustrate the proposed methods with some examples taken from the literature on general equilibrium models.
\end{abstract}

Key words: general equilibrium, computation of equilibria, interior-point methods

\section{Introduction}

The field of computational economics has undergone a revolution as a consequence of the recent advances in computational science. Nowadays economists use a wide range of methods to compute equilibria in increasingly large and complicated economic models. In practice, the criteria for choosing between these computational methods should be based on efficiency and accuracy.

Computational methods for finding equilibria can require too much computer time or storage, as economic models can be extremely large and nonlinear. Inefficient algorithms may make it difficult to solve an economic model. Moreover, the success of a computational approach depends on its accuracy. An algorithm should be able to identify a solution with precision, reducing the rounding errors that occur when the algorithm is implement on a computer.

In this paper, we consider two ways for characterizing equilibria. The first characterization uses the excess demand function, whilst the second one is based on first order conditions of the consumers' problems, the firms' problems and the market clearing conditions that define the economic model. Both characterizations can be seen as a nonlinear complementarity problem (NLCP).

The main aim of this paper is to present an alternative algorithm for solving NLCP based on interior-point methods. In particular, we propose to solve an NLCP as a least-squares problem. The use of interior-point methods avoids one of the weaknesses of the least-squares approach, namely, the ill-conditioning problem 
often observed. Moreover, as in the Gauss-Newton method, our algorithm will exploit the special structure of the problem, omitting the second order information of the system of equations to solve. This is a great advantage in terms of cost.

Since the 1980s, interior-point methods have become popular for solving nonlinear constrained problems in an efficient and practical manner even for largescale problems, but they have not had a similar echo in the field of Computational Economics. Although some skill is required in choosing appropriate parameters, interior-point methods have proven to be an efficient alternative for solving non-linear constrained problems (for a discussion, see Morales et al., 2001).

A second objective is to survey the main approaches for computing equilibria in general equilibrium models and indicate how some of these methods can be improved from the computational point of view.

We illustrate the proposed approach with some examples taken from the literature on general equilibrium models. These examples show how the proposed interior-point approach can find accurate solutions with little computational cost.

The rest of the paper is organized as follows. In Section 2 we give a formal description of the basic model and present the concept of equilibrium. Section 3 is devoted to briefly review the literature on computing general equilibria. Using the excess demand function, an equilibrium can be the solution of an NLCP. In Section 4, we present an efficient algorithm to solve NLCP based on interior-point methods. Instead of using the excess demand function, in Section 5 we propose to compute the equilibrium values as the solution of an alternative system of equations and some simple bounds, using the algorithm presented in Section 4 based on interior-point methods.

\section{Competitive General Equilibrium Model}

In this section we first present the Competitive General Equilibrium equilibrium model. This model was introduced by Walras (1874), and later it was formalized by Arrow and Debreu in this century (see e.g., Arrow and Hahn, 1971; Debreu, 1959).

We consider an economy with $D$ perfectly divisible commodities, $I$ consumers and $J$ firms. We assume that all relevant information is available when agents take decisions (i.e., perfect information). We take $\mathbb{R}^{D}$ as the commodity space. Each commodity $d=1, \ldots, D$ will have associated with it a real number $p_{d}$ representing its price. In what follows, $x^{T}$ denotes the transpose of $x \in \mathbb{R}^{D}$, a $D$ dimensional row vector. For any $x, y \in \mathbb{R}^{D}, x \cdot y=x^{T} y$ denotes the inner product of vectors $x$ and $y$.

We assume that the $j$-th firm maximizes an objective function (e.g., its profit) on its production set $\mathbb{Y}_{j} \subset \mathbb{R}^{D}$. A production set for the $j$-th firm is a set composed of all production plans which are possible for such a firm, according to the technological knowledge available. A production set $\mathbb{Y}_{j}$ is commonly described 
by means of a set of functions $F_{j}: \mathbb{R}^{D} \rightarrow \mathbb{R}^{K_{j}}$, called transformation functions, $\mathbb{Y}_{j}=\left\{y_{j} \in \mathbb{R}^{D}: F_{j}\left(y_{j}\right) \leq 0\right\}$.

Usually, general equilibrium models assume that the firm's objective is to maximize its profit, i.e., $o_{j}\left(y_{j}, p\right)=p \cdot y_{j}$. However, in the real world the objectives of the firm result from the objectives of those individuals who control it. For instance, a firm might consider maximizing the expected profit, or a firm could be interested in the maximization of its minimum profit because of its aversion to risk, or the maximization of the short or long-term sales revenues, or the size of the firm's labor force (for example, in the case of a cooperative). Therefore, their objectives may be different, and the profit maximization assumption does not apply to them. To encompass different decision rules, we define the objective function of the $j$-th firm as a function $o_{j}: \mathbb{Y}_{j} \times \mathbb{R}^{D} \rightarrow \mathbb{R}$, where $\mathbb{Y}_{j}$ is the production set and $\mathbb{R}^{D}$ is the price space. For each firm $j \in\{1, \ldots, J\}$, the function $o_{j}\left(y_{j}, p\right)$ is homogeneous of degree $\alpha$ in prices $p$, for some $\alpha>0$. Thus, given a price vector $p \in \mathbb{R}^{D}$ and a production set $\mathbb{Y}_{j} \subset \mathbb{R}^{D}$, the $j$-th firm faces the problem

$$
\max _{y_{j} \in \mathbb{Y}_{j}}\left\{o_{j}\left(y_{j}, p\right): p \cdot y_{j} \geq 0\right\} .
$$

When firms are profit maximizers and have the possibility of inaction $\left(0 \in \mathbb{Y}_{j}\right)$, there is no need to take into account the no-loss constraints $\left(p \cdot y_{j} \geq 0\right)$. On the other hand, this constraint is not too restrictive. For example, consider a static and stochastic economy with a commodity that can be traded at $S$ states of the world. Even if we require $p \cdot y=\sum_{s=1}^{S} p_{s} \cdot y_{s} \geq 0$, we are still allowing $p_{s} \cdot y_{s}<0$ for a particular state of the world $s$. In an intertemporal and deterministic economy, for some period of time $t_{0}$ it may hold that $p_{t_{o}} \cdot y_{t_{o}}<0$.

Under convexity and compactness assumptions, it is possible to prove the existence of a solution for this problem. This solution, known as the $j$-th firm supply, is a correspondence denoted by $\hat{y}_{j}(p)$. Furthermore, under strong convexity, such a solution is a function. Finally, note that $\hat{y}_{j}(p)$ is homogeneous of degree zero whenever we assume $o_{j}\left(y_{j}, p\right)$ to be homogeneous in prices. In particular, $\hat{y}_{j}(p)$ is homogeneous of degree zero if we assume that firms are profit maximizers.

On the other hand, the choice set for the $i$-th consumer is given by a subset $\mathbb{X}_{i} \subset \mathbb{R}^{D}$ which describes feasible consumption vectors. We will assume that the consumption set of the $i$-th consumer is described by inequality constraints $\mathbb{X}_{i}=\left\{x_{i} \in \mathbb{R}^{D}: G_{i}\left(x_{i}\right) \leq 0\right\}$. Each consumer has preferences given by a utility function $u_{i}: \mathbb{X}_{i} \rightarrow \mathbb{R}$, and is endowed with a vector $w_{i} \in \mathbb{X}_{i}$. Assuming private ownership market economies (the consumers own the firms), $r_{i j} \in \mathbb{R}_{+}$ denotes the $i$-th consumer's participation in the profit of the $j$-th firm, with $\sum_{i=1}^{I} r_{i j}=1, \forall j=1, \ldots, J$. Given a price vector $p \in \mathbb{R}^{D}$, the $i$-th consumer's demand can be expressed as the solution to the following program:

$$
\max _{x_{i} \in \mathbb{X}_{i}}\left\{u_{i}\left(x_{i}\right): p \cdot x_{i} \leq p \cdot w_{i}+\sum_{j=1}^{J} r_{i j} p \cdot y_{j}\right\} \text {. }
$$


Under convexity and compactness assumptions, it is possible to prove the existence of a solution for this problem. This solution, known as the $i$-th consumer demand, is a correspondence that we will denote by $\hat{x}_{i}(p)$. Furthermore, under strong convexity, such a solution is a function. Finally, note that $\hat{x}_{i}(p)$ is homogeneous of degree zero.

An economy can thus be described by a set

$$
E=\left(\left\{\mathbb{X}_{i}, u_{i}\right\}_{i=1}^{I},\left\{\mathbb{Y}_{j}, o_{j}\right\}_{j=1}^{J},\left\{w_{i}\right\}_{i=1}^{I},\left\{r_{i j}\right\}_{i, j}\right) .
$$

Next, we introduce the concept of equilibrium for an economy $E$, and review the main results on existence of equilibria.

DEFINITION 1. Competitive equilibrium. The vector prices $p^{*} \in \mathbb{R}^{D}$, with $p^{*} \neq 0$, and the allocation $\left(x^{*}, y^{*}\right) \in \prod_{i=1}^{I} \mathbb{X}_{i} \times \prod_{j=1}^{J} \mathbb{Y}_{j}$ is an equilibrium for an economy $E$, if $x_{i}^{*}$ solves the decision problem of the $i$-th consumer (2); $y_{j}^{*}$ solves the decision problem of the $j$-th firm (1); and it is satisfied the market clearing condition: $\sum_{i=1}^{I} x_{i}^{*}=\sum_{j=1}^{J} y_{j}^{*}+\sum_{i=1}^{I} w_{i}$.

As a consequence of the homogeneity of degree zero of the demand and supply functions in the prices, without loss of generality, we can define prices on the positive simplex

$$
\Delta_{p}^{+}=\left\{p \in \mathbb{R}^{D}: \sum_{d=1}^{D} p_{d}=1, p_{d} \geq 0, d=1, \ldots, D\right\} .
$$

The excess demand function for an economy $E$ is defined as

$$
z(p)=\sum_{i=1}^{I} \hat{x}_{i}(p)-\sum_{j=1}^{J} \hat{y}_{j}(p)-\sum_{i=1}^{I} w_{i} .
$$

In this setting, any price $p^{*} \gg 0$ is an equilibrium price if and only if $z\left(p^{*}\right)=0$. Therefore, $p^{*}$ corresponds to the solution of a system of nonlinear equations. In general, we can define prices on

$$
\Delta_{p}=\left\{p \in \mathbb{R}^{D}: \sum_{d=1}^{D}\left|p_{d}\right| \leq 1\right\}
$$

and in this case, with locally nonsatiated preferences, a price $p^{*} \in \mathbb{R}_{+}^{D}$, with $p^{*} \neq 0$ is an equilibrium price if and only if

$$
z\left(p^{*}\right) \leq 0, \quad p^{*} \geq 0, \quad p^{*} \cdot z\left(p^{*}\right)=0 .
$$

This expression says that all goods in excess supply have a zero price. The condition $p^{*} \cdot z\left(p^{*}\right)=0$ is known as Walras' law, see e.g., Arrow and Hahn (1971). 
Alternatively, equilibria prices $p^{*} \in \Delta_{p}^{+}$can be characterized as a solution of the variational inequality

$$
z\left(p^{*}\right)\left(p-p^{*}\right) \leq 0, \quad \forall p \in \Delta_{p}^{+},
$$

whenever the function $z$ satisfies Walras' law. For details see Ch. 7, Nagurney (1993).

In Optimization literature, Problem (3) is known as the basic NonLineal Complementarity Problem (NLCP) and Problem (4) is known as the basic Variational Inequality Problem (VIP). Many results for NLCP can be applied to VIP. The survey in Harker and Pang (1990) provides an excellent introduction to these problems.

The study of the existence of equilibria has played an important role in the development of computational procedures. The existence proofs are often constructive, and therefore, suggests algorithms for computing equilibria. Most of the recent literature on existence of Walrasian equilibria is based on fixed point theorems, following Arrow and Debreu (1954). The idea is to find a fixed point $p^{*}$ of a continuous correspondence $q$ such that $z\left(p^{*}\right)=0$ if and only if $p^{*} \in q\left(p^{*}\right)$.

To compute an equilibrium, it is necessary to state practical conditions that characterize these points. The traditional literature on computing equilibria uses the excess demand function. The main disadvantage of this approach is that equilibria may be difficult to compute since, in large applied models, it is difficult to specify the functional form of excess demand functions. However, there exists an alternative approach to avoid to compute the excess demand function.

Negishi (1960) presented an alternative proof of the existence of equilibria based on mathematical programming techniques. Assuming that firms are profit maximizers, he proved that a competitive equilibrium is a maximum point of a social welfare function which is a linear combination of utility functions of consumers subject to all the market clearing conditions. In other words, an equilibrium $\left(x^{*}=x\left(\delta^{*}\right), y^{*}=y\left(\delta^{*}\right), p^{*}=\lambda\left(\delta^{*}\right)\right)$ is the solution of the master problem

$$
\max _{(x, y) \in \prod_{i=1}^{I} \mathbb{X}_{i} \times \prod_{j=1}^{J} \mathbb{Y}_{j}}\left\{\sum_{i=1}^{I} \delta_{i} u_{i}\left(x_{i}\right): \sum_{i=1}^{I} x_{i} \leq \sum_{j=1}^{J} y_{j}+\sum_{i=1}^{I} w_{i}\right\},
$$

for a certain $\delta^{*}$ such that $\delta^{*} \gg 0$ and transfers

$$
t_{i}\left(\delta^{*}\right)=\lambda\left(\delta^{*}\right) \cdot\left(x_{i}\left(\delta^{*}\right)-w_{i}-\sum_{j=1}^{J} r_{i j} y_{j}\left(\delta^{*}\right)\right)=0 .
$$

Takayama and El Hodiri (1968) and Diewert (1970) also provided a similar result based on these techniques. A demonstration of the existence of equilibrium when firms have a general objective to maximize can be found in Esteban-Bravo (2000). 
Therefore, under convexity assumptions, an equilibrium $\left(x^{*}, y^{*}, \lambda^{*}\right)$ is characterized by the first order conditions of the master problem and transfers equal to zero, with $\delta_{i}^{*} \gg 0, \forall i=1, \ldots, I$. In other words, an equilibrium $\left(x^{*}, y^{*}, \lambda^{*}\right)$ is characterized by the first order conditions of the consumers' problems and the firms' problems and the market clearing conditions that define the economic model:

$$
\begin{aligned}
& \delta_{i}^{*} \nabla_{x_{i}} u_{i}\left(x_{i}^{*}\right)-\lambda^{* T}-v_{i}^{*} \nabla_{x_{i}} G_{i}\left(x_{i}^{*}\right)=0, \forall i=1, \ldots, I, \\
& \nabla_{y_{j}} o_{j}\left(y_{j}^{*}, \lambda^{*}\right)-\xi_{j}^{*} \lambda^{* T}-\mu_{j}^{*} \nabla_{y_{j}} F_{j}\left(y_{j}^{*}\right)=0, \forall j=1, \ldots, J, \\
& v_{i}^{*} G_{i}\left(x_{i}^{*}\right)=0, G_{i}\left(x_{i}^{*}\right) \geq 0, \forall i=1, \ldots, I, \\
& \mu_{j}^{*} F_{j}\left(y_{j}^{*}\right)=0, F_{j}\left(y_{j}^{*}\right) \leq 0, \forall j=1, \ldots, J, \\
& \xi_{j}^{*}\left(\lambda^{* T} y_{j}^{*}\right)=0, \lambda^{* T} y_{j}^{*} \geq 0, \forall j=1, \ldots, J, \\
& \lambda^{* T}\left(x_{i}^{*}-w_{i}-\sum_{j=1}^{J} r_{i j} y_{j}^{*}\right)=0, \forall i=1, \ldots, I, \\
& \sum_{+}^{I} i=1 x_{i}^{*}=\sum_{j=1}^{J} y_{j}^{*}+\sum_{i=1}^{I} w_{i} ;
\end{aligned}
$$

with $\delta_{i}^{*} \gg 0, \forall i=1, \ldots, I$. See Judd (1999, pp. 189-190). A further discussion of this issue can be found in Esteban-Bravo (2000).

The characterization (5) is described by inequality constraints that can be transformed into equations by adding nonnegative slack variables. Therefore, an equilibrium is characterized by equality constraints and bound constraints. Let $H(z)=0$ denote the system (5) of nonlinear equations that characterize an equilibrium, where $z$ now contains both the variables and the slacks. Also, let $l \leq z \leq u$ denote the bound constraints, where $l$ and $u$ are vectors of lower and upper bounds on the components of $z$. Some components of $z$ may lack a lower or an upper bound, in these cases we set the appropriate components of $l$ and $u$ to $-\infty$ and $+\infty$, respectively. In Section 5 we outline how an equilibrium can be computed using this characterization that we call Welfare Characterization.

\section{Standard Numerical Methods for Computing Equilibria}

Now, we briefly review the currently available algorithms for computing equilibria.

\subsection{SIMPLICIAL METHODS}

These methods are algorithms for computing fixed points of a continuous mapping of the simplex $\Delta_{p}^{+}$into itself, based on the constructive proof of the Brouwer fixed point theorem (1912). The customary proof of Brouwer's theorem makes use of the combinatorial result known as Sperner's lemma (1928). The use of some variant of Brouwer's fixed point theorem is an essential step to prove the existence of equilibria in game theory and general equilibrium theory.

Applications of simplicial methods to general equilibrium theory are originally due to Scarf $(1967,1982,1998)$. Following Debreu (1959), he defined a continuous 
mapping from the price simplex into itself, derived from the excess market demand function for the given economy. Scarf's method computes the fixed points of this mapping, $p^{*} \in \Delta_{p}^{+}$, that are the equilibrium prices, using the algorithm proposed by Lemke and Howson (1964) and Lemke (1965). The main contribution of Scarf's method is the use of a new combinatorial argument, more practical and efficient than Sperner's lemma from the computational point of view (Scarf, 1967, 1982, 1998; Scarf and Hansen, 1973).

The main criticism of these methods is that they are at best linearly convergent. Subsequent contributions have refined Scarf's algorithm in terms of flexibility and speed of convergence. For example, Hansen (1968) and Kuhn $(1968,1969)$ improve Scarf's algorithm by giving a more efficient procedure to exchange vertices of simplices.

In the context of NLCP, this approach is known as projection-type methods and was proposed by Cottle (1966). The linearization methods, studied originally by Mathiesen (1985), compute the equilibrium by solving a sequence of linear complementarity problems using Lemke's algorithm. However this approach is only appropriate for providing local convergence. In 1995, Dirkse and Ferris proposed an algorithm with global convergence properties via reformulation of the problem as a nonsmooth system of equations. This algorithm is basically based on a nonsmooth Newton method proposed by Robinson (1994). Subsequent contributions have refined this approach and been implemented in the PATH solver, see for example Ferris et al. (1999). The main difficulty associated to this solver is that some skill is required in choosing an approriate initial point. This is due to the fact that this Newton-based solver uses an active set approach, and it is well known that errors in the active constraints have large effect on the accuracy of the solution (see Fletcher, 1987, for example). Although heuristic techniques to identify an appropriate active set are used (see, for example, Dirkse and Ferris, 1997), these algorithms may fail to find the solution.

\subsection{TÂTONNEMENT APPROACH}

Since Samuelson $(1941,1942,1947)$ first formalized the concept of tâtonnement, algorithms based on this idea have become very popular. The tâtonnement theory describes the process of price adjustments based on the discrepancy between supply and demand.

The intuition suggests that prices will adjust upward for goods in excess demand and downward for those in excess supply. Therefore, the tâtonnement process can be described by a continuous dynamic system of the form $\dot{p}_{t}=z\left(p_{t}\right)$, or alternatively, by the discrete analogous of the form $\Delta p_{t}=z\left(p_{t-1}\right)$. When it is assumed that prices cannot be negative, this process is described by $\dot{p}_{t}=G\left(z\left(p_{t}\right)\right)$ or alternatively, its discrete analogue $p_{t-1}-p_{t}=G\left(z\left(p_{t-1}\right)\right)$, where $G(\cdot)$ is a function such that $G(\cdot) \geq 0$ (see examples of functional forms of $G$ in Arrow and Hahn (1983)). 
The dynamic behavior of both systems is analogous: both of them achieve the same equilibria or stationary states, possess the same type of stability and rates of convergence or divergence to equilibria are equivalent. As we are interested in just finding equilibria (no trajectory to this equilibrium has a realistic economic interpretation), equilibrium prices $p^{*}$ can be obtained by means of the continuous or discrete system (see, e.g., Luenberger, 1979). Even though it is easier to solve the discrete system than the continuous one, the literature on tâtonnement algorithms usually consider the continous version.

\subsection{PATH FOLLOWING OR HOMOTOPY METHODS}

Continuation methods are techniques for numerically approximating a solution curve which is implicitly defined by an undetermined system of equations. The basic idea of these algorithms is to solve 'easy' problems that gradually are transformed into the original problem. In other words, to solve $p-f(p)=0$, a map $H(p, t)$ is chosen such that $H(p, t)=0$ is trivial to solve at $t=0$ and $H(p, t)=p-f(p)=0$ at $t=1$, which means we solve the original problem. The function $H$ is known as a homotopy and the sequence of solutions $\left\{p_{t}\right\}$ from $t=0$ to $t=1$ is known as the path.

These methods rely on a certain regularity condition: the Jacobian $\nabla_{p} H(\cdot, t)$ has full rank along the path $\left\{p_{t}\right\}$. Suppose that we want to solve $H(x)=0$, where $x=(p, t)$. Differentiation of $H(x)=0$ yields $D$ conditions on the $D+1$ unknowns, $\nabla H(x) \dot{x}=0$. This system has an infinite number of solutions such as $\dot{x}_{i}=(-1)^{D-i+1} \operatorname{det}\left(\nabla H(x)_{i}\right)$, where $\nabla H(x)_{i}$ means we remove the $i$-th column of $\nabla H(x)$. Therefore, if $\nabla H(x)$ is nonsingular, this differential equation leads to a solution of $H(x)=0$. Thus, to find a zero of $H(x)$ we need to solve a system of differential equations. These differential equations are typically solved using numerical techniques such as Runge-Kutta methods which suggests that these methods are of limited practical use. Note that, for example, the Runge-Kutta method of order four requires the calculation of four matrix inversions per iteration in contrast to the Newton's method that just requires one matrix inversion.

In the context of computation of equilibria, the use of homotopies in simplicial algorithms to approximate fixed points was introduced by Eaves (1972), Merril (1972), and Van der Laan and Talman (1979). Much recent work on the computation of fixed points has been based on the continuation, path following or homotopy methods. See Eaves and Schmedders (1999). However, as Harker and Pang (1990, p. 162) pointed out, these methods can be inappropriate from the practical point of view due to the difficulty with solving medium to large scale equilibrium models. Note that the computation or approximation of Jacobians will become increasingly impractical as the problem size increases. Furthermore, these methods may fail to compute a solution even to a quite simple set of nonlinear equations (see Example 11.2, Nocedal and Wright, 1999). 


\subsection{NEWTON METHOD}

The most reasonable method to solve a system of nonlinear continuously differentiable equations $z(p)=0$ is the use of a Newton-like algorithm. The $k$-th iteration of a Newton-like method can be written as $p_{k}=p_{k-1}+\alpha d$, where $\alpha=1$ for the standard Newton method and $d$ is given by $\nabla z\left(p_{k-1}\right) d=-z\left(p_{k-1}\right)$. Its main feature is the potential for a much more rapid rate of convergence. However, the Newton method only ensures local convergence. Alternatives to assure global convergence are line search methods, trust-region methods and filter-type methods (this one is very promising but too recent). For an introduction, see Nocedal and Wright (1999), for example.

Although there are approaches to calculate the derivatives automatically such as automatic differentiation and symbolic differentiation techniques (see Chapter 7 , Nocedal and Wright, 1999, for further details), unfortunately there are many instances where the computation of the exact formula of the Jacobian $\nabla z(p)$ is a very expensive and difficult task. In this case, quasi-Newton methods (and particularly limited-memory quasi-Newton methods) are an efficient way to avoid the computation of $\nabla z$ while preserving reasonable local convergence properties, see Nocedal and Wright (1999) for example. Moreover, it is worthwhile using methods that exploit the structure of $\nabla z$ in order to reduce the requirements of computation and storage to compute the direction of search $d$. For details, see Dennis and Schnabel (1996).

The use of Newton method to compute equilibria is not a common practice in the literature of computable general equilibrium, although the Newton method is more efficient than the methods presented in previous sections. This literature points out that this method can present problems due to the behaviour of the Jacobian of the excess demand function, $\nabla z\left(p^{*}\right)$ (see, e.g., Ginsburgh and Keyzer, 1997, pp. 14-15). As a consequence of the aggregation, the excess demand function $z(p)$ may lose most of the favorable regularity properties that characterize the individual supply and demand functions. In particular, the Jacobian $\nabla z(p)$ could be singular or, being regular, the eigenvalues of its inverse could be close to being zero. Even if the behavior of the matrix $\nabla z(p)$ is adequate, it is possible that $\nabla z(p)$ violates the nonsingularity condition at points along the sequence of points generated by the algorithm.

None of these problems essentially affects the convergence of the algorithm. In fact, a large number of authors recommend the use of Newton method since it often yields rapid solutions in practice (see, e.g., Whalley, 1973; Ginsburgh and Waelbroeck, 1981; Adelman and Robinson, 1978, among others).

\subsection{SMALE'S METHOD}

An alternative algorithm for computing fixed points was developed by Smale (1974-1976, 1982). Similarly to Scarf's method, he showed the existence of solutions for the excess demand equations using Brouwer's theorem, but based on 
different calculus. The algorithm proposed by Smale is based on a version of Brouwer's theorem, proposed by Hirsch (1963). A similar approach had been suggested by Kellog, Li and Yorke (1976).

Let $B$ be the unit disk $B=\left\{p \in \mathbb{R}^{D}: p^{T} p \leq 1\right\}$, and let $g$ be a continuous differentiable map $g: B \rightarrow B$. Smale proposed starting with a regular value $(p-g(p))$, a point $\bar{p} \in \partial B$, and then, solving $\lambda\left(p_{t}\right)\left(p_{t}-g\left(p_{t}\right)\right)=\bar{p}$, as the solution of this functional equation must terminate at a fixed point. Differentiating the previous equation with respect to $t$,

$$
\begin{aligned}
& \lambda\left(p_{t}\right)\left(I-\nabla g\left(p_{t}\right)\right) \dot{p}_{t}+\nabla \lambda\left(p_{t}\right)\left(p_{t}-g\left(p_{t}\right)\right)=0 \Leftrightarrow \\
& \left(I-\nabla g\left(p_{t}\right)\right) \dot{p}_{t}=-\mu\left(p_{t}\right)\left(p_{t}-g\left(p_{t}\right)\right),
\end{aligned}
$$

where $\mu\left(p_{t}\right)=\nabla \lambda\left(p_{t}\right) / \lambda\left(p_{t}\right)$. Then, the equilibrium $p^{*}$ is the solution of this system of differential equations. Unfortunately, the computation of solutions for this type of problems may be very difficult in practice, and requires the use of numerical techniques for solving systems of differential equations.

\section{Computation of Equilibria as an NLCP via Interior-point Methods}

As discussed before, an equilibrium can be characterized by the excess demand function. Then, an equilibrium can be seen as the solution of an NLCP, that is, to find a vector $p^{*} \in \mathbb{R}^{D}$ such that

$$
z\left(p^{*}\right) \leq 0, \quad p^{*} \geq 0, \quad p^{*} \cdot z\left(p^{*}\right)=0 .
$$

An NLCP is described by inequalities that can be transformed into equations by adding nonnegative slack variables, $s$, as follows

$$
z(p)+s=0, \quad p^{T} s=0,
$$

and certain bound constraints $(p, s) \geq 0$. Assuming that $z: \mathbb{R}^{D} \rightarrow \mathbb{R}^{D}$ is continuously differentiable everywhere, the interior-point standard approach (see Wright, 1997, pp. 167-168), considers the perturbed system of equations $z\left(p_{k}\right)+s_{k}=0$, $P_{k} s_{k}-\mu_{k} e=0$ to compute the solution of the original problem $\mu_{k} \rightarrow 0$, where $e$ is the unit vector and $P_{k}=\operatorname{diag}\left(p_{k}\right)$. However, this procedure is not always but the recommendable one due to ill-conditioning problems as it was pointed out by Gill et al. (1981, pp. 139-140).

The algorithm that we present in this paper differs from that in that it adopts a least squares approach. In the proposed approach the ill-conditioning problems seem to be less harmful than in the standard. We formulate Problem (7) as a boundconstrained least squares problem:

$$
\min \left\{\frac{1}{2}\left\|\left(\begin{array}{c}
z(p)+s \\
p^{T} s
\end{array}\right)\right\|_{2}^{2},(p, s) \geq 0\right\} .
$$


Note that a vector $p^{*} \in \mathbb{R}^{D}$ solves the Problem (6) if and only if $p^{*} \in \mathbb{R}^{D}$ solves the Problem (8) for some $s^{*} \geq 0$ and $z\left(p^{*}\right)+s^{*}=0, p^{* T} s^{*}=0$. To prove this characterization of the Problem (6) is straightforward.

The methodology for solving this problem is closely related to that of the nonlinear programming problems. The implementation of interior-point methods lead to solve Problem (8) by formulating a sequence of unconstrained subproblems defined as

$$
\min \frac{1}{2}\left\|\left(\begin{array}{c}
z(p)+s \\
p^{T} s-\mu e
\end{array}\right)\right\|_{2}^{2}-\mu \sum_{i=1}^{D} \log p_{i}-\mu \sum_{i=1}^{D} \log s_{i} .
$$

A detailed description of the proposed algorithm can be found in Appendix A.

One of the main features of our algorithm is that it exploits the special structure of the problem as in the Gauss-Newton method, omitting the second order information of $z(p)+s=0$, what is clearly more economical in terms of function evaluations. Moreover, the use of interior-point methods avoids one of the weaknesses of the least-squares approach, namely, the ill-conditioning problem often observed as the Newton direction $(\Delta p, \Delta s)^{T}$ is computed from

$$
\left(\begin{array}{cc}
J\left(p_{k}, s_{k}\right)^{T} J\left(p_{k}, s_{k}\right) & I \\
\left(W_{k}^{1} 0\right) & P_{k} \\
\left(0 W_{k}^{2}\right) & S_{k}
\end{array}\right)\left(\begin{array}{c}
\Delta p \\
\Delta s \\
\Delta w^{1} \\
\Delta w^{2}
\end{array}\right)=-\left(\begin{array}{c}
J\left(p_{k}, s_{k}\right)^{T} H\left(p_{k}, s_{k}\right)-\left(\begin{array}{c}
w_{k}^{1} \\
w_{k}^{2}
\end{array}\right) \\
P_{k} W_{k}^{1}-\mu_{k} e \\
S_{k} W_{k}^{2}-\mu_{k} e
\end{array}\right),
$$

where $J\left(p_{k}, s_{k}\right)$ denotes the Jacobian matrix of $H\left(p_{k}, s_{k}\right)=\left(\begin{array}{c}z\left(p_{k}+s_{k}\right. \\ P_{k} s_{k}\end{array}\right)$, $w_{k}^{1}=\mu_{k} p_{k}^{-1}, w_{k}^{2}=\mu_{k} p_{k}^{-1}$, and $\mu_{k}=p_{k}^{T} s_{k} / D$. Another important feature of this method is that the major computational effort in each step is the computation of the Newton's direction $(\Delta p, \Delta s)^{T}$ (i.e., to solve a linear system of equations). A main advantage of this approach is that whenever $\nabla z\left(p_{k}\right)$ has full rank, the Newton's direction is a suitable direction for Problem (9). Therefore, given careful attention how to solve this system of equations, this algorithm has a great potential for the computation of equilibria in large-scale models. There is a large literature that studies how to solve very large linear systems. An excellent book in this area is Golub and van Loan (1996) (see also Byrd, Hribar and Nocedal, 1999).

We now present some examples of economic models that can be found in the recent literature on economic applications of NLCP. These examples illustrate the proposed approach, that could be easily extended to larger economic models. As we will see, this approach successfully computes the equilibria in only few iterations. Therefore this approach appears to be better both in terms of speed and reliability.

EXAMPLE 2. Static exchange economy (Kehoe, 1991, pp. 2066-2067). Consider a static exchange economy with two consumers and two goods. The $i$-th consumer has a utility function of the form $u_{i}\left(x_{1}, x_{2}\right)=\sum_{j=1}^{2} a_{i j}\left(x_{j}^{b_{j}}-1\right) / b_{j}$, 
where $a_{i j}=1024$ if $i=j, a_{i j}=1$ if $i \neq j, b_{j}=-4$ and initial endowments $w_{i j}=12$ if $i=j, w_{i j}=1$ if $i \neq j, \forall i, j=1,2$. Taking as the initial point $z_{0}=1^{T}$, the algorithm converges to

$$
z^{*}=[0.9518,0.0482,0.0002,0.0025]^{T}
$$

in 13 steps. Hence, the equilibrium prices are $p^{*}=(0.9518,0.0482)^{T}$.

EXAMPLE 3. Production economy (Kehoe, 1991, pp. 2083-2084). Consider a production economy with two consumers and four goods. The $i$-th consumer has a utility function of the form $u_{i}\left(x_{1}, x_{2}\right)=\sum_{j=1}^{4} a_{i j} \log x_{j}$ and $w_{i}$ initial endowments. His demand function is

$$
x_{j}^{i}\left(p_{1}, p_{2}\right)=\frac{a_{i j} \sum_{k=1}^{4} p_{k} w_{i k}}{p_{j} \sum_{k=1}^{4} a_{i k}}, \forall i, j=1,2,
$$

where

$$
a=\left(\begin{array}{cccc}
0.8 & 0.2 & 0 & 0 \\
0.1 & 0.9 & 0 & 0
\end{array}\right), \quad w=\left(\begin{array}{cccc}
0 & 0 & 10 & 0 \\
0 & 0 & 0 & 20
\end{array}\right) .
$$

The production technology is specified by a $4 \times 8$ activity matrix

$$
A=\left[\begin{array}{cccccccc}
-1 & 0 & 0 & 0 & 3 & 5 & -1 & -1 \\
0 & -1 & 0 & 0 & -1 & -1 & 5 & 5 \\
0 & 0 & -1 & 0 & -1 & -1 & -1 & -4 \\
0 & 0 & 0 & -1 & -1 & -4 & -3 & -1
\end{array}\right]
$$

Taking as the initial point $z_{0}=1^{T}$, the algorithm converges to the equilibrium prices $p^{*}=(0.25,0.22,0.36,0.16)^{T}$ and the equilibrium production plan $y^{*}=$ $(0,0,0,0,5.18,0.36,4,45,0)^{T}$, in 24 steps.

EXAMPLE 4. Mathiesen (1987, pp. 5-6). Consider a static production economy with one consumer, one producer and three goods. The consumer has a demand function of the form

$$
d_{l}\left(x_{1}, x_{2}, x_{3}\right)=\frac{\alpha_{i}\left(b_{2} p_{2}+b_{3} p_{3}\right)}{p_{l}}, \forall l=1,2,3,
$$

and initial endowment $b=\left(b_{1}, b_{2}, b_{3}\right)^{T}$. The firm has a technology matrix $A=$ $[1,-1,-1]^{T}$. Taking $\alpha=0.9$ and $b=(0,5,3)^{T}$, for example, and given the initial point $z_{0}=1^{T}$, the algorithm converges to $z^{*}=[0.5,0.08,0.41,3,0.15,0.05$, $0.01,0.03,0,0,0,0]^{T}$ in 16 steps. Hence, in equilibrium, the production for the firm is $y^{*}=3$, with the associated normalized price $p^{*}=(0.5,0.08,0.41)^{T}$. 
EXAMPLE 5. Pure exchange model (Scarf and Hansen, 1973, Ch.3). Consider a static production economy with five consumers and ten goods. Each consumer $i$-th has an initial endowment $w_{i}$. The consumer has a demand function of the form

$$
d_{i}(\pi)=\frac{\alpha_{i} \pi \cdot w_{i}}{\pi_{i}^{\beta_{i}} \sum_{k} \alpha_{i k} \pi_{k}^{1-\beta_{i}}},
$$

where $\alpha_{i k}$ is the demand share parameter for consumer $i$ and good $k$, and $\beta_{i}$ is the elasticity of substitution for consumer $i$. Taking

$$
\alpha=\left(\begin{array}{cccccccccc}
1 & 1 & 3 & 0.1 & 0.1 & 1.2 & 2 & 1 & 1 & 0.7 \\
1 & 1 & 1 & 1 & 1 & 1 & 1 & 1 & 1 & 1 \\
9.9 & 0.1 & 5 & 0.2 & 6 & 0.2 & 8 & 1 & 1 & 0.2 \\
1 & 2 & 3 & 4 & 5 & 6 & 7 & 8 & 9 & 10 \\
1 & 13 & 11 & 9 & 4 & 0.9 & 8 & 1 & 2 & 10
\end{array}\right)
$$

$b=(2,1.3,3,0.2,0.6)^{T}$, and

$$
w=\left(\begin{array}{cccccccccc}
0.6 & 0.2 & 0.2 & 20 & 0.1 & 2 & 9 & 5 & 5 & 15 \\
0.2 & 11 & 12 & 13 & 14 & 15 & 16 & 5 & 5 & 9 \\
0.4 & 9 & 8 & 7 & 6 & 5 & 4 & 5 & 7 & 12 \\
1 & 5 & 5 & 5 & 5 & 5 & 5 & 8 & 3 & 17 \\
8 & 1 & 22 & 10 & 0.3 & 0.9 & 5.1 & 0.1 & 6.2 & 11
\end{array}\right) .
$$

for example, and given the initial point $z_{0}=1^{T}$, the interior-point algorithm converges to the equilibrium prices

$$
p^{*}=(0.18,0.11,0.1,0.04,0.11,0.07,0.11,0.1,0.09,0.4)^{T}
$$

in 30 steps.

EXAMPLE 6. A production economy (Scarf and Hansen, 1973, Ch. 5). Consider a static production economy with six consumers, eight activity sectors and six goods. Each consumer $i$-th has an initial endowment $w_{i}$. The consumer has a demand function of the form

$$
d_{i}(\pi)=\frac{\alpha_{i} \pi \cdot w_{i}}{\pi_{i}^{\beta_{i}} \sum_{k} \alpha_{k} \pi_{k}^{1-\beta}},
$$

where $\alpha_{i}$ is the demand share parameter for consumer $i$, and $\beta_{i}$ is the elasticity of substitution for consumer $i$. The firm has a technology matrix $A=[-I ; B]$, where

$$
B=\left[\begin{array}{cccccccc}
4 & 4 & 1.6 & 1.6 & 1.6 & 0.9 & 7 & 8 \\
-5.3 & -5 & -2 & -2 & -2 & -1 & -4 & -5 \\
-2 & -1 & -2 & -4 & -1 & 0 & -3 & -2 \\
-1 & -6 & -3 & -1 & -8 & 0 & -1 & -8 \\
0 & 0 & 6 & 8 & 7 & 0 & 0 & 0 \\
4 & 3.5 & 0 & 0 & 0 & 0 & 0 & 0
\end{array}\right]
$$


Taking

$$
\alpha=\left(\begin{array}{cccccc}
4 & 0 & 0.2 & 0 & 2 & 3.2 \\
0.4 & 0 & 0 & 0.6 & 4 & 1 \\
2 & 0 & 0.5 & 0 & 2 & 1.5 \\
5 & 0 & 0 & 0.2 & 5 & 4.5 \\
3 & 0 & 0 & 0.2 & 4 & 2
\end{array}\right), \quad w=\left(\begin{array}{cccccc}
0 & 3 & 5 & 0.1 & 0 & 1 \\
0 & 0.1 & 0.1 & 7 & 0 & 2 \\
0 & 2.0 & 6 & 0.1 & 0 & 1.5 \\
0 & 1 & 0.1 & 8 & 0 & 1 \\
0 & 6 & 0.1 & 0.5 & 0 & 2
\end{array}\right),
$$

and $b=(1.2,1.6,0.8,0.5,0.6)$ for example, and given the initial point $z_{0}=1^{T}$, the interior-point algorithm converges to the equilibrium prices $p^{*}=(0.22,0.25$, $0.16,0.05,0.1,0.2)^{T}$ and the equilibrium production plan $y^{*}=(0,0,0,0,0,0$, $0.46,0,3.93,0,0,0,0.43,0)^{T}$ in 24 steps.

EXAMPLE 7. A production economy (Scarf and Hansen, 1973, Ch. 5). Consider a static production economy with four consumers, twenty-six activity sectors and fourteen goods. Each consumer $i$-th has an initial endowment $w_{i}$. The consumer has a demand function of the form $d_{i}(\pi)=\alpha_{i} \pi \cdot w_{i} / \pi_{i}$, where $\alpha_{i}$ is the demand share parameter for consumer $i$. The activity analysis matrix and the parameters $\alpha$ and $w$ are given in Scarf and Hansen (1973, pp. 115-116). Then, given the initial point $z_{0}=1^{T}$, the interior-point algorithm converges to the interior-point algorithm converges to the equilibrium prices

$$
\begin{aligned}
p^{*}= & (0.062,0.058,0.095,0.071,0.065,0.062,0.068,0.098,0.09, \\
& 0.079,0.056,0.062,0.036,0.092)^{T}
\end{aligned}
$$

and the equilibrium production plan

$$
\begin{aligned}
y^{*}= & (\underset{1 \times 14}{\mathbf{0}}, 4.79, \underset{1 \times 2}{\mathbf{0}}, 51.9,4.04, \underset{1 \times 3}{\mathbf{0}}, 30.5,21.1,36.8 \\
& 28.02,0,44.04,23.6,0,25.6,0,12.05, \underset{1 \times 3}{\mathbf{0}}, 47.2, \underset{1 \times 3}{\mathbf{0}})^{T}
\end{aligned}
$$

in 28 steps.

Finally, we study how an equilibrium can be computed as a solution of a variational inequality problem (VIP). The generalization of the standard nonlinear complementarity problem (3) is the mixed complementarity problem (MCP). Given a function $z: \mathbb{R}^{D} \rightarrow \mathbb{R}^{D}$, the MCP is to find $p^{*} \in \mathbb{R}^{D}, w^{*} \in \mathbb{R}_{+}^{D}$ and $v^{*} \in \mathbb{R}_{+}^{D}$ such that

$$
z\left(p^{*}\right)=w^{*}-v^{*}, l \leq p^{*} \leq u,\left(p^{*}-l\right)^{T} w^{*}=0,\left(u-p^{*}\right)^{T} v^{*}=0,
$$

where $l$ and $u$ denote vectors of lower and upper bounds on the components of $x$, respectively. As before, this Problem (10) can be solved by means of the equivalent inequality-constrained optimization problem:

$$
\min \left\{\frac{1}{2}\left\|\left(\begin{array}{c}
z(p)-w+v \\
(p-l)^{T} w \\
(u-p)^{T} v
\end{array}\right)\right\|_{2}^{2}: l \leq p \leq u, 0 \leq w, 0 \leq v\right\},
$$


using the proposed algorithm. This extension is specially useful since the mixed complementarity problem formulation includes many standard problems such as zero-finding for a system of nonlinear or linear equations, the nonlinear or linear complementarity problems, nonlinear problems and the finite-dimensional systems of variational inequalities.

In general, a VIP over a nonempty subset $X \subseteq \mathbb{R}^{D}$ consists of finding $p^{*} \in \mathbb{R}^{D}$ such that $z\left(p^{*}\right)^{T}\left(p-p^{*}\right) \leq 0, \forall p \in X$. Note that when $X=\mathbb{R}_{+}^{D}$, the Problem VIP reduces to NLCP, and when $X=[l, u]$, the Problem VIP becomes the MCP. Therefore, whenever the computation of equilibria is formulated as a VIP, the proposed approach can also solve this type of problems in an efficient manner.

However, from the economic point of view, there are no advantages of using this characterization instead of the one that characterizes an equilibrium as a solution of a NLCP (neither when demonstrating the existence of the equilibrium nor when calculating these equilibria).

\section{Computation of Equilibria Using the Welfare Characterization via Interior-point Methods}

Previously, we have presented an efficient algorithm based on the excess demand function. But, as we mentioned before, it can be quite difficult to obtain its functional form in general. Mantel (1971) first considered the use of Negishi's approach to compute equilibria. He proposed a tâtonnement algorithm to solve the continuous version $\dot{\delta}_{t}=-t\left(\delta_{t}\right)$. However, as we have outlined before, this procedure is only useful for certain simple economies due to the numerical difficulties of solving differential equations.

In this section we presented an alternative algorithm to compute a competitive equilibrium using the Welfare Characterization in an efficient and practical manner via interior-point methods. We also present some examples to illustrate the proposed approach, that could be easily extended to larger economic models.

We consider the system of nonlinear equations (5), $H(z)=0$, where $z \in \mathbb{R}^{D *}$ and the bound constraints are of the form $l \leq z \leq u$. This problem can be seen as an NLC problem. Therefore, our approach will follow the scheme used in Section 4, considering the alternative inequality-constrained optimization problem:

$$
\min _{l \leq z \leq u} \frac{1}{2}\|H(z)\|_{2}^{2} .
$$

See Appendix A for details.

We now present some examples of economic models that can be found in the recent literature on computation of general equilibria. These examples illustrate the proposed approach, that could be easily extended to larger economic models.

EXAMPLE 8. Static exchange economy (Kehoe, 1991, pp. 2066-2067). Consider the exchange economy with two consumers and two goods described in Exam- 
ple 2. Taking as the initial point $z_{0}=[0.5,0.5,12,1,1,12,0.5,0.5]^{T}$, the interiorpoint algorithm converges to $z^{*}=[1.0992,1.0992,10.39,2.6,2.6,10.39,0.0093$, $0.0093]^{T}$ in 7 steps. Note that the major computational effort in each step is the computation of the Newton direction $\left(\Delta z, \Delta w^{1}, \Delta w^{2}\right)$; i.e., to solve a linear system of equations. Hence, in equilibrium, the consumption for consumers 1 and 2 is $x_{1}^{*}=(10.39,2.6)^{T}$ and $x_{2}^{*}=(2.6,10.39)^{T}$, respectively, with the associated price $p^{*}=(0.0093,0.0093)^{T}$.

EXAMPLE 9. Static production economy I. Consider a static production economy with one consumer, one producer and two goods. The consumer has a utility function of the form $u\left(x_{1}, x_{2}\right)=x_{2}-\left(-x_{1}+4\right)^{2}$, and an initial endowment $w=(5,15)$. The firm has a production set $Y=\left\{\left(y_{1}, y_{2}\right): y_{2}=16-\left(y_{1}+4\right)^{2}\right\}$. Given the initial point $z_{0}=[1,6,20,1,5,3,1,1]^{T}$, the interior-point algorithm converges to

$$
z^{*}=[2.31,2.5,28.75,-2.5,13.75,6.93,2.31,2.31]^{T}
$$

in 6 steps. Hence, in equilibrium, the consumption for the consumer is $x^{*}=$ $(2.5,28.75)^{T}$ and the production for the firm is $y^{*}=(-2.5,13.75)^{T}$, with the associated price $p^{*}=(2.31,2.31)^{T}$.

EXAMPLE 10. Static production economy II. Consider a static production economy with two consumers, two producers and two goods. Consumers have utility functions of the form $u_{1}\left(x_{11}, x_{12}\right)=x_{12}-\left(-x_{11}+4\right)^{2}$, and $u_{2}\left(x_{21}, x_{22}\right)=$ $x_{22}-\left(-x_{21}+6\right)^{2}$, and initial endowments $w_{1}=(5,15), w_{2}=(15,5)$. Firms have production sets $Y_{1}=\left\{\left(y_{11}, y_{12}\right): y_{12}=16-\left(y_{11}+4\right)^{2}\right\}$, and $Y_{2}=\left\{\left(y_{21}, y_{22}\right): y_{22}=-5 y_{11}\right\}$. Given the initial point $z_{0}=[1,1,5,15,15$, $5,0.5,0.5,0.5,0.5,5,1,1,1]^{T}$, the interior-point algorithm converges to $z^{*}=$ $[1.7,8.5,1.5,34.75,14.82,5.9,-1.5,9.75,-2.18,10.9,8.5,1.7,1.7,1.7]^{T}$ in 9 steps. Hence, in equilibrium, the consumption for consumers 1 and 2 is $x_{1}^{*}=$ $(1.5,34.75)^{T}$ and $x_{2}^{*}=(14.82,5.9)^{T}$, respectively, the production for firms 1 and 2 is $y_{1}^{*}=(-1.5,9.75)^{T}$ and $y_{2}^{*}=(-2.18,10.9)^{T}$, respectively, with the associated price $p^{*}=(1.7,1.7)^{T}$.

EXAMPLE 11. Static production economy II. Consider a static production economy with one consumer, two producers and two goods. The consumer has a utility function of the form $u\left(x_{1}, x_{2}\right)=x_{2}-\left(-x_{1}+4\right)^{2}$, and $w=(5,10)$. Firms have production sets $Y_{1}=\left\{\left(y_{11}, y_{12}\right): y_{12}=16-\left(y_{11}+4\right)^{2}\right\}$ and $Y_{2}=\left\{\left(y_{21}, y_{22}\right) ; y_{22}=36-\left(y_{21}+6\right)^{2}\right\}$. Given the initial point $z_{0}=$ $[1,1,31,10,16,10,5,5,1,1,1]^{T}$, the interior-point algorithm converges to $z^{*}=$ $[1.84,1,44,-1,7,-3,27,91.9233,1.84,1.84,1.84]^{T}$ in 7 steps. Hence, in equilibrium, the consumption for the consumer is $x^{*}=(1,44)^{T}$, the production for firms 1 and 2 is $y_{1}^{*}=(-1,7)^{T}$ and $y_{2}^{*}=(-3,27)^{T}$, respectively, with the associated price $p^{*}=(1.84,1.84)^{T}$. 
EXAMPLE 12. Static production economy IV. Consider the following static production economy. Suppose that the firms' objectives are the following: $o_{1}\left(y_{1}, p\right)=\frac{1}{3} p_{1}^{T} y_{11}+\frac{2}{3} p_{2}^{T} y_{12}$ and $o_{2}\left(y_{2}, p\right)=\frac{2}{3} p_{1}^{T} y_{21}+\frac{1}{3} p_{2}^{T} y_{22}$. Given the initial point

$$
z_{0}=[1,1,31,10,16,10,5,5,1,1,1]^{T},
$$

the interior-point algorithm converges to

$$
z^{*}=[1.88,1.42,33.89,-2.71,14.34,-0.85,9.55,55.58,1.88,1.25,0.62]^{T}
$$

in 7 steps. Hence, in equilibrium, the consumption for the consumer is $x^{*}=$ $(1.42,33.89)^{T}$, the production for firms 1 and 2 is $y_{1}^{*}=(-2.71,14.34)^{T}$ and $y_{2}^{*}=(-0.85,9.55)^{T}$, respectively, with the associated price $p^{*}=(1.25,0.62)^{T}$.

EXAMPLE 13. Static production economy V. Consider a static production economy with one consumer, two producers and two goods. The consumer has a utility function of the form $u\left(x_{1}, x_{2}\right)=x_{2}-\left(-x_{1}+4\right)^{2}$, and initial endowment $w=(5,10)$. Firms have production sets

$$
\begin{aligned}
& Y_{1}=\left\{\left(y_{11}, y_{12}\right): y_{12}=16-\left(y_{11}+4\right)^{2}\right\}, \\
& Y_{2}=\left\{\left(y_{21}, y_{22}\right): y_{22}=36-\left(y_{21}+6\right)^{2}\right\}, \\
& Y_{3}=\left\{\left(y_{31}, y_{32}\right): y_{32}=5 y_{31}\right\} .
\end{aligned}
$$

Given the initial point $z_{0}=[1,1,31,10,16,10,5,1,1,5,1,1,1,1]^{T}$, the interior-point algorithm converges to

$$
\begin{aligned}
z^{*}= & {[1.7,1.5,42,-1.5,9.75,-3.5,29.75,1.5,-7.5,50.9,1.7,-1.7,} \\
& -1.7,-1.7]^{T}
\end{aligned}
$$

in 8 steps. Hence, in equilibrium, the consumption for the consumer is $x^{*}=$ $(1.5,42)^{T}$, the production for firms 1,2 and 3 is $y_{1}^{*}=(-1.5,9.75)^{T}, y_{2}^{*}=$ $(-3.5,29.75)^{T}$ and $y_{3}^{*}=(1.5,-7.5)^{T}$, respectively, with the associated price $p^{*}=(-1.7,-1.7)^{T}$.

EXAMPLE 14. Static production economy VI with the present of externalities. Consider a static production economy with one consumer, two producers $(j=1,2)$ and two goods $(l=1,2)$. Assume that there are two externalities affecting firm 2: one generated by consumer's consumption of good 1 and one generated by production of good 1 by firm 1 . This can be the case when there exist pollution of a river by city inhabitants (municipal sewage) and a firm (industrial effluents) that affect a downstream water-using firm.

The consumer has a utility function of the form $u\left(x_{1}, x_{2}\right)=x_{2}-\left(-x_{1}+4\right)^{2}$, and an initial endowment $w=(5,10)$. Firms have production sets

$$
\begin{aligned}
& Y_{1}=\left\{\left(y_{11}, y_{12}\right): y_{12}=16-\left(y_{11}+4\right)^{2}\right\} \\
& Y_{2}=\left\{\left(y_{21}, y_{22}\right): y_{22}=36-y_{11}-\left(y_{21}+6+x_{1}\right)^{2}\right\}
\end{aligned}
$$


Given the initial point $z_{0}=[1,1,31,10,16,10,5,5,1,1,1]^{T}$, the interiorpoint algorithm converges to $z^{*}=[78.34,0.75,41.62,-0.75,5.43,-3.5,26.18$, $509.23,78.34,78.34,78.34]^{T}$, in 17 steps. Hence, in equilibrium, the consumption for the consumer is $x^{*}=(0.75,41.62)^{T}$, the production for firms 1 and 2 is $y_{1}^{*}=(-0.75,5.43)^{T}$ and $y_{2}^{*}=(-3.5,26.18)^{T}$, respectively, with the associated price $p^{*}=(509.23,78.34)^{T}$.

Effectively firm 2 is adversely affected by the consumer and the firm 1 as the profits of firm 1 and 2 in the presence of externalities are less than in the absence of them (see Example III that assumes absence of any externalities).

\section{EXAMPLE 15. A production model for the deregulated electricity market} with externalities. Consider a static production economy for electric power producers in a deregulated market. In recent years, the ongoing liberalization process in the electricity markets has created a significant interest in the development of economic models that may represent their behavior. Modeling the technologies of a generation company requires a special attention. Generators decide about production under the restriction of their technological knowledge and in the transmission network.

We consider two consumers $(i=1,2)$, four producers $(j=1,2,3,4)$ and four goods $(l=1,2,3,4)$. Assume that Firms 1,2 and 3 produce electricity using labor and fuel as inputs, and Firm 4 produces a non-energetic commodity, which can be read as a non-energetic industrial production index. Furthermore, we assume that there are network capacity constraints affecting electric power firms 1, 2, 3, and therefore the total amount of electricity that these firms can produce will be bound by the network externalities. On the other hand, we assume that consumers' utilities do not depend on fuel, are positively affected by the energetic and non-energetic commodities, and negatively by work. Moreover, Consumer 1 is endowed with fuel and labor, whilst Consumer 2 possesses participations in all firms' profits.

Let $x_{1 i}, y_{1 j}$ denote the consumption and production of non-energetic commodities, respectively; $x_{2 i}, y_{2 j}$ denote the consumption and production of electricity, respectively; $y_{3 j}$ denote the demand of fuel; and $x_{4 i}, y_{4 j}$ denote the labor offer and demand, respectively. Each commodity will have associated with it a real nonnegative number $p_{l}$ representing its price.

Consumer 1 has a utility function of the Leontief form

$$
u\left(x_{11}, x_{21}, x_{31}, x_{41}\right)=\left(x_{11}^{-\rho}+a x_{21}^{-\rho}\right)^{-1 / \rho},
$$

where $\rho=0.5, a=0.10248384$, and an initial endowment $w=(250,0,721,0)$. We assume that this consumer does not work $\left(x_{41}=0\right)$ as he/she owns all the firms. Thus, the consumer 1 faces the problem

$$
\begin{array}{ll}
\max & \left(x_{11}^{-\rho}+a x_{21}^{-\rho}\right)^{-1 / \rho} \\
\text { s.a. } & p_{1} x_{11}+p_{2} x_{21} \leq p_{1} w_{11}+p_{3} w_{31}+B(p, y), \\
& x_{11}, x_{21} \geq 0
\end{array}
$$


where

$$
\begin{aligned}
B(p, y)= & p_{1} y_{14}+p_{2}\left(y_{21}+y_{22}+y_{23}-y_{24}\right) \\
& -p_{3}\left(y_{31}+y_{32}+y_{33}\right)-p_{4}\left(y_{41}+y_{42}+y_{43}+y_{44}\right) .
\end{aligned}
$$

Consumer 2 has a Cobb-Douglas utility function, i.e.,

$$
u\left(x_{12}, x_{22}, x_{32}, x_{42}\right)=x_{12}^{d_{1}} x_{22}^{d_{2}}\left(w_{42}-x_{42}\right)^{d_{4}},
$$

where $d_{1}=0.5, d_{2}=0.11093647, d_{4}=0.11969204$, and an initial endowment $w=(350,0,420,4000)$. Thus, the consumer 2 faces the problem

$$
\begin{array}{ll}
\max & x_{12}^{d_{1}} x_{22}^{d_{2}}\left(w_{42}-x_{42}\right)^{d_{4}} \\
\text { s.a. } & p_{1} x_{12}+p_{2} x_{22}-p_{4} x_{42} \leq p_{1} w_{12}+p_{3} w_{32} \\
& x_{12}, x_{22}, x_{42} \geq 0, x_{42} \leq w_{42} .
\end{array}
$$

We assume that all firms maximize their profits on their production set described by means of a Cobb-Douglas production function. The network externalities affecting firms 1, 2, 3 are given by the constraint $y_{21}+y_{22}+y_{23} \leq 250$, therefore, each $j$-th firm's problem can be expressed by the following program:

$$
\begin{array}{ll}
\max & p_{2} y_{2 j}-p_{3} y_{3 j}-p_{4} y_{4 j} \\
\text { s.a. } & y_{21}+y_{22}+y_{23} \leq 250 \\
& y_{2 j} \leq A_{j}\left(y_{3 j}\right)^{a_{j}}\left(y_{4 j}\right)^{b_{j}} \\
& 0 \leq y_{2 j} \leq K_{j}, y_{3 j}, y_{4 j} \geq 0,
\end{array}
$$

for all $j=1,2,3$, where $A_{1}=2, a_{1}=1 / 2, b_{1}=1 / 3, K_{1}=200$; and $A_{2}=$ 1.409567, $a_{2}=1 / 3, b_{2}=1 / 3, K_{2}=150$, and $A_{3}=0.6350529, a_{3}=1 / 3$, $b_{3}=1 / 2, K_{3}=180$.

On the other hand, the 4-th firm faces the following problem:

$$
\begin{array}{ll}
\max & p_{1} y_{14}-p_{2} y_{24}-p_{4} y_{44} \\
\text { s.a. } & y_{14} \leq A_{4}\left(y_{24}\right)^{a_{4}}\left(y_{44}\right)^{b_{4}}, \\
& y_{24}, y_{44} \geq 0,
\end{array}
$$

where $A_{4}=3.535938, a_{4}=1 / 4, b_{4}=1 / 2$.

Finally, note that the market clearing conditions are given as follows:

$$
\begin{aligned}
x_{11}+x_{12} & =y_{14}+w_{11}+w_{12}, \\
x_{21}+x_{22} & =y_{21}+y_{22}+y_{23}+y_{24}, \\
0 & =y_{31}+y_{32}+y_{33}+w_{31}+w_{32}, \\
x_{42} & =y_{41}+y_{42}+y_{43}+y_{44}+w_{41}+w_{42} .
\end{aligned}
$$


Thus, given the initial point $z_{0}=1^{T}$, the interior-point algorithm converges to the equilibrium

$$
\begin{aligned}
& x_{1}^{*}=(617.47,9.66,0,0)^{T} \\
& x_{2}^{*}=(528.35,70.854,0,2784.026)^{T}, \\
& y_{1}^{*}=(0,199.9,653.35,59.8)^{T}, \\
& y_{2}^{*}=(0,0,0.01,0.002)^{T}, \\
& y_{3}^{*}=(0,50.089,487.63,100.42)^{T}, \\
& y_{4}^{*}=(545.82,82.47,0,2623.8)^{T}, \\
& p^{*}=(1,1.65,0.014,0.104)^{T},
\end{aligned}
$$

in 360 steps. It is important to point out that the proposed algorithm obtains solutions in a few seconds. Note that the major computational effort in each step is the calculation of the Newton direction; i.e., to solve a linear system of equations.

This example could be easily extended by considering that all the goods are indexed by a geographical location on a network. In such a case, the capacity bounds of the transport lines would be local between each two nodes of the electric network. This is the case of electric markets in which coordination between different electric power stations is required to improve the efficient use of resources because electricity cannot be stored and requires immediate delivery.

The same arguments can be applied in transport economic models, communication models, and related spatial economic models where congestion effects play a relevant role. This type of economic models can be extremely large and nonlinear, and therefore economists require the use of efficient numerical methods to compute equilibria, as the algorithm proposed.

\section{Summary and Conclusions}

We have seen many different methods that are available for the computation of equilibria in general equilibrium models. We have also investigated any further improvements in the efficiency of the existing algorithms. When the functional form of the excess demand function is available, Newton-type methods are recommended due to their potential for a rapid rate of convergence. However, this approach cannot deal with simple bounds, and therefore, it cannot be applied to economic models with nonnegative prices.

In this paper we have presented a new approach to solve nonlinear complementarity problems as a least-squares problem via interior-point methods. This methodology has better efficiency than the standard approach (see Wright, 1997, pp. 167-168) that fails to compute a solution even to quite simple examples. This is mainly due to the fact that ill-conditioning problems in the proposed approach seems to be less harmful than in the standard one. Moreover, as in the GaussNewton method, our algorithm will exploit the special structure of the problem, 
omitting the second order information of the system of equations to solve. This is a great advantage in terms of cost. The proposed method has been tested on several examples that can be found in the recent literature on computable general equilibrium. The results show that this approach works quite well and could be easily extended to larger economic models due to their practicability.

The recent literature on computation of general equilibria uses the excess demand function, but in applied models it is difficult to specify the functional form of the demand and supply functions. The use of the welfare approach avoids this difficulty as equilibria are characterized by the first order conditions of the consumers' problems and the firms' problems and the market clearing conditions that define the economic model. Using this characterization, we have proposed an algorithm to compute equilibria as a least-squares problem via interior-point methods. The practicability of the welfare approach and the efficiency of the proposed algorithm applied to this characterization can be shown complex economies such as those that present externalities.

\section{Acknowledgements}

This paper contains part of the author's doctoral dissertation written at Universidad Carlos III de Madrid, Spain, under the supervision of Prof. F.J. Prieto. I wish to thank Prof. F.J. Prieto for his help and encouragement in the direction of my thesis and in the preparation of this paper. I am also grateful to Prof. M. Florenzano and Prof. P. Gourdel for their advice and encouragement since the very early stage of my thesis. I also thank Prof. B. Rusten and an anonymous referee for the careful reading of the paper and constructive comments that have led to an improved version of the paper.

These research has been supported by a Marie Curie Fellowship of the European Community programme 'Improving Human Research Potential and the Socio-economic Knowledge Base' under contract number HPMF-CT-2000-00781.

\section{Appendix A: Interior Point Methods}

Interior point methods are closely related to the classical logarithmic barrier methods. The barrier method is defined by introducing a parameter $\mu$, called barrier parameter, and a logarithmic barrier function that is defined in the interior of the feasible set of Problem (11). Problem (11) can then be solved by formulating a sequence of uncontrained subproblems defined as:

$$
\min _{z} \frac{1}{2}\|H(z)\|_{2}^{2}-\mu \sum_{i=1}^{I} \log \left(z_{i}-l_{i}\right)-\mu \sum_{i=1}^{*} \log \left(u_{i}-z_{i}\right) .
$$

Under mild conditions, every limit point of a sequence $\left\{z^{*}(\mu)\right\}$ of local minimizers of Problem (12) is a local minimum of the original constrained Problem (11); i.e., 
$z^{*}(\mu) \underset{\mu \rightarrow 0}{\longrightarrow} z^{*}$. This method was studied by Fiacco and McCormick (1968). For an introduction to interior-point methods see, e.g., Wright (1991) and its references, and for details, see Nesterov and Nemirovskii (1994).

To apply an interior-point method to Problem (2) we use the first-order conditions:

$$
J\left(z_{k}\right)^{T} H\left(z_{k}\right)-\mu\left(Z_{k}-L\right)^{-1}+\mu\left(U-Z_{k}\right)^{-1}=0,
$$

where $Z_{k}=\operatorname{diag}\left(z_{k}\right), L=\operatorname{diag}(l), U=\operatorname{diag}(u)$ and $J\left(z_{k}\right)$ denotes the Jacobian matrix of $H$. Let $W_{k}^{1}=\mu\left(Z_{k}-L\right)^{-1}$ and $W_{k}^{2}=\mu\left(U-Z_{k}\right)^{-1}$, then we can rewrite the first-order conditions as

$$
\begin{aligned}
& J\left(z_{k}\right)^{T} H\left(z_{k}\right)-w_{k}^{1}+w_{k}^{2}=0, \\
& \left(Z_{k}-L\right) W_{k}^{1}-\mu=0, \\
& \left(U-Z_{k}\right) W_{k}^{2}-\mu=0 .
\end{aligned}
$$

We will denote this system as $F\left(z_{k}, w_{k}^{1}, w_{k}^{2}\right)=0$, where $w_{k}^{1}, w_{k}^{2} \geq 0$. This system is the standard primal-dual system slightly modified since we omit the second order information of $H(z)$ as Gauss-Newton method. This is the system that we will solve using Newton algorithm (see e.g., Dennis and Schnabel, 1996, pp. 86-154).

Step 1. Let $z_{0}, w_{0}^{1}, w_{0}^{2}$ and $\varepsilon>0$. Set $k=1, z_{k} \leftarrow z_{0}, w_{k}^{1} \leftarrow w_{0}^{1}$, and $w_{k}^{2} \leftarrow w_{0}^{2}$.

Step 2. If $\left\|F\left(z_{k}, w_{k}^{1}, w_{k}^{2}\right)\right\|_{2}<\varepsilon$, stop (the problem is solved); else, solve the system

$$
\left(\begin{array}{ccc}
J\left(z_{k}\right)^{T} J\left(z_{k}\right) & -I & I \\
W_{k}^{1} & \left(Z_{k}-L\right) & 0 \\
-W_{k}^{2} & 0 & \left(U-Z_{k}\right)
\end{array}\right)\left(\begin{array}{c}
\Delta z \\
\Delta w^{1} \\
\Delta w^{2}
\end{array}\right)=-F\left(z_{k}, w_{k}^{1}, w_{k}^{2}\right)
$$

Step 3. Compute $\alpha_{z}, \alpha_{w^{1}}, \alpha_{w^{2}} \in(0,1)$ such that $z_{k+1}=z_{k}+\alpha_{z} \Delta z, w_{k+1}^{1}=w_{k}^{1}+$ $\alpha_{w^{1}} \Delta w^{1}$ and $w_{k+1}^{2}=w_{k}^{2}+\alpha_{w^{2}} \Delta w^{2}$ are feasible.

Step 4. Consider the merit function

$$
M(z ; \mu)=\frac{1}{2}\|H(z)\|_{2}^{2}-\mu \sum_{i=1}^{I} \log \left(z_{i}-l_{i}\right)-\mu \sum_{i=1}^{I} \log \left(u_{i}-z_{i}\right),
$$

and let $m(\alpha)=M(z+\alpha \Delta z ; \mu)$.

While $m(0)-m\left(\alpha_{z}\right)<-\rho \alpha_{z} \nabla m(0)^{T} \Delta z$, where $0<\rho<1$; set $\alpha_{z} \leftarrow \alpha_{z} / 2$ and $z_{k+1}=z_{k}+\alpha_{z} \Delta z$. 


$$
\mu \leftarrow \gamma \frac{\left(z_{k}-l\right)^{T} w_{k}^{1}+\left(u-z_{k}\right)^{T} w_{k}^{2}}{2 D^{*}},
$$

where $0 \leq \gamma<1$, and $k \leftarrow k+1$ and go back to step 2 .

Step 3 ensures strict feasibility with respect to the bound constaints (in this context, $\delta_{i}>0, \forall i=1, \ldots, I$, and $w>0$ ). The parameter $\mu$ is updated in terms of the average value of the complementarity products $\left(z_{k}-l\right)^{T} w_{k}^{1}$ and $\left(u-z_{k}\right)^{T} w_{k}^{2}$. The success of this algorithm depends critically on the choice of the parameters $\mu$ and $\gamma$. Unfortunately, difficulties can arise if unsuitable values of these parameters are used (see e.g., Wright, 1991).

\section{References}

Adelman, I. and Robinson, S. (1978). Income Distribution Policy in Developing Countries: A Case Study of Korea. Stanford University Press, Stanford, CA.

Arrow, K.J. and Debreu, G. (1954). Existence of equilibrium for a competitive economy. Econometrica, 22, 265-290.

Arrow, K.J. and Hahn, F.H. (1971, 1983). General Competitive Analysis. Advanced Textbooks in Economics, Vol. 12. North Holland, New York.

Brouwer, L.E. (1912). Über Abbildung von Manningfaltigkeiten. Math. Ann., 71, 97-115.

Byrd, R.H., Hribar, M.E. and Nocedal, J. (1999). An interior point algorithm for large-scale nonlinear programming. SIAM Journal on Optimization, 9(4), 877-900.

Cottle, R.W. (1966). Nonlinear programs with positively bounded jacobians. SIAM Journal on Applied Mathematics, 14, 147-158.

Debreu, G. (1959, 1987). Theory of Value. In 1959, John Wiley and Sons, New York. Second edition in 1987, Cowless Foundation Monographs, 17, Yale University Press, New Haven.

Dennis, J.E. Jr. and Schnabel, R.B. (1996). Numerical Methods for Unconstrained Optimization and Nonlinear Equations. SIAM, Philadelphia.

Diewert, W.E. (1970). On a theorem of Negishi. Metroeconomica, XXV, 119-135.

Dirkse, S.P. and Ferris, M.C. (1995). The PATH solver: A non-monotone stabilization scheme for mixed complementarity problems. Optimization Methods and Software, 5, 123-156.

Dirkse, S.P. and Ferris, M.C. (1997). Crash techniques for large-scale complementarity problems. In M.C. Ferris and J.S. Pang (eds.), Complementarity and Variational Problems: State of the Art. SIAM Publications, Philadelphia, Pennsylvania.

Eaves, B.C. (1972). Homotopies for the computation of fixed points. Mathematical Pogramming, 3, 225-237.

Eaves, B.C. and Schmedders, K. (1999). General equilibrium models and homotopy methods. Journal of Economic Dynamics and Control, 23, 1249-1279.

Esteban-Bravo, M. (2000). Existence and computation of equilibria in general equilibrium. Ph.D. thesis, Universidad Carlos III de Madrid, Spain, December.

Ferris, M.C., Kanzow, C. and Munson, T. (1999). Feasible descent algorithms for mixed complementarity problems. Mathematical Programming, 86, 475-497.

Fiacco, A.V. and McCormick, G.P. (1968). Nonlinear Programming: Sequential Unconstrained Minimization Techniques. John Wiley and Sons, New York.

Fletcher, R. (1987). Practical Methods of Optimization, 2nd edn. Wiley, Chichester.

Gale, D. (1955). The law of supply and demand. Mathematica Scandinavia, 3, 155-169. 
Garcia, C.B. and Zangwill, W.I. (1981). Pathways to Solutions, Fixed Points, and Equilibria. Prentice Hall, Englewood Cliffs, NJ.

Gill, P.E., Murray, W., Sauders, M.A., Tomlin, J.A. and Wright, M.H. (1986). On projected Newton barrier methods for linear programming and an equivalence to Karmarkar's projective method. Mathematical Programming, 36, 183-209.

Ginsburgh, V. and Keyzer, M. (1997). The Structure of Applied General Equilibrium Models. The MIT Press, Cambridge.

Ginsburgh, V. and Waelbroeck, J.L. (1981). Activity Analysis and General Equilibrium Modelling. North-Holland, Amsterdam.

Golub, G.H. and Van Loan, C.F. (1996). Matrix Computations, 3rd edn. The Johns Hopkins University Press, Baltimore.

Hansen, T. (1968). On the approximation of a competitive equilibrium. Ph.D. thesis, Yale University.

Harker, P.T. and Pang, J.S. (1990). Finite-dimensional variational inequality and nonlinear complementarity problems: a survey of theory, algorithms and applications. Mathematical Programming, 48, 161-220.

Hirsch, M.W. (1963). A proof of the nonretractability of a cell into its boundary. Proceedings of the American Mathematical Society, 14, 364-365.

Judd, K.L. (1998). Numerical Methods in Economics. The MIT Press, Cambridge.

Kehoe, T.J. (1991). Computation and multiplicity of equilibria. In W. Hildenbrand and H. Sonnenschein (eds.), Handbook of Mathematical Economics, Vol. IV, Ch. 38, 2049-2144. Elsevier Science Publishers B.V.

Kellog, R.B., Li, T.Y. and Yorke, J. (1976). A constructive proof of the Brouwer fixed-point theorem and computational results. SIAM Journal of Numerical Analysis, 13, 478-483.

Kuhn, H.W. (1968). Simplicial approximation of fixed points. Proc. Nat. Acad. Sci., 61, 1238-1242.

Kuhn, H.W. (1969). Approximate search for fixed points. In Computing Methods in Optimization Problems-2. Academic Press, New York.

Lemke, C.E. (1965). Bi-matrix equilibrium points and mathematical programing. Management Science, 11, 681-689.

Lemke, C.E. and Howson, J.T. (1964). Equilibrium points of bi-matrix games. SIAM Journal of Applied Mathematics, 12, 412-423.

Luenberger, D.G. (1979). Introduction to Dynamic Systems: Theory, Models, and Applications. Wiley, New York.

Mantel, R.R. (1971). The welfare adjustment process: its stability properties. International Economic Review, 12, 415-430.

Mathiesen, L. (1985). Computational experience in solving equilibrium models by a sequence of linear complementarity problems. Operations Research, 33(6), 1225-1250.

Merril, O.H. (1972). Applications and extension of an algorithm that computes fixed points of certain upper semi-continuous point to set mappings. Ph.D. thesis, Department of Industrial Engineering, University of Michigan.

McKenzie, L. (1959). On the existence of general equilibrium for a competitive market. Econometrica, 27, 54-71.

Morales, J.L., Waltz, R., Liu, G., Goux, J.P. and Nocedal, J. (2001). Assessing the potential of interior methods for nonlinear optimization. Proceedings of the first Sandia workshop on large-scale PDE-constrained optimization. In O. Ghattas (ed.), Springer's Lecture Notes in Computational Science and Engineering. Santa Fe, New Mexico, U.S.A.

Nagurney, A. (1993). Network Economies - A Variational Inequality Approach. Kluwer Academic Publishers, Dordrecht, The Netherlands.

Negishi, T. (1960). Welfare economics and the existence of an equilibrium for a competitive economy. Metroeconomica, XII, 92-97.

Nikaido, H. (1956). On the classical multilateral exchange problem. Metroeconomica, VIII, 135145. 
Nesterov, Y. and Nemirovskii, A. (1994). Interior-Point Polynomial Algorithms in Convex Programming. SIAM, Philadelphia.

Nocedal, J. and Wright, S.J. (1999). Numerical Optimization. Springer Series in Operations Research. Springer-Verlag, New York.

Ortega, J.M. and Rheinboldt, W.C. (1970). Iterative Solution of Nonlinear Equations in Several Variables. Academic Press, San Diego.

Robinson, S.M. (1994). Newton's method for a class of nonsmooth functions. Set-Valued Analysis, 2, 291-305.

Samuelson, P.A. (1941). The stability of equilibrium: comparative statics and dynamics. Econometrica, 9, 97-120.

Samuelson, P.A. (1942). The stability of equilibrium: linear and nonlinear systems. Econometrica, 10, 1-25.

Samuelson, P.A. (1947). Foundations of Economic Analysis. Harvard University Press, Cambridge, Mass.

Scarf, H.E. (1967). The approximation of fixed points of a continuous mapping. Journal of Applied Mathematics, 15, 1328-1343.

Scarf, H.E. (1982). The computation of equilibrium prices: an exposition. In W. Hildenbrand and H. Sonnenschein (eds.), Handbook of Mathematical Economics, Vol. II, Ch. 21, 1007-1061. Elsevier Science Publishers B.V.

Scarf, H.E. (1998). The computation of equilibrium prices. In A. Kirman (ed.), Elements of General Equilibrium Analysis, Ch. 4, 88-138. Blackwell Publishers Ltd.

Scarf, H.E. and Hansen, T. (1973). The Computation of Economic Equilibria. Yale University Press, New Haven.

Smale, S. (1974-76). Global analysis and economics, IIA-VI. Journal of Mathematical Economics, 1, 1-14, 107-117, 119-127, 213-221.

Smale, S. (1976). A convergent process of price adjustment and global Newton methods. Journal of Mathematical Economics, 3, 1-14.

Smale, S. (1982). Global analysis and economics. In W. Hildenbrand and H. Sonnenschein (eds.), Handbook of Mathematical Economics, Vol. I, Ch. 8, 331-370. Elsevier Science Publishers B.V.

Sperner, E. (1928). Neuer Beweis für die Invarianz der Dimensionszahl und des Gebietes. Abh. a.d. Math. Sem. d. Univ. Hamburg, 6, 265-272.

Takayama, A. and El Hodiri, M. (1968). Programming, Pareto optimum and the existence of competitive equilibria. Metroeconomica, XX, 1-10.

Van der Laan, G. and Talman, A.J.J. (1979). A restart algorithm for computing fixed points without an extra dimension. Mathematical Programming, 17, 74-84.

Walras, L. (1874, 1954). Eléments d'économie politique pure. In 1874, L. Corbaz, Lausanne. English translation of William Jaffé in 1954: Elements of Pure Economics. Allen and Unwin, London.

Whalley, J. (1973). A numerical assessment of the 1973 United Kingdom. Ph.D. thesis, Yale University.

Wright, M.H. (1991). Interior methods for constrained optimization. Acta Numerica, 341-407.

Wright, S. (1997). Primal-Dual Interior-Point Methods. SIAM Philadelphia, PA. 